\title{
1 The Genetic Architecture of Larval Aggregation Behavior in \\ 2 Drosophila
}

3 Ross M McKinney and Yehuda Ben-Shahar ${ }^{\mathrm{a}}$

$4 \quad{ }^{a}$ Department of Biology, Washington University in St. Louis, St. Louis, MO, USA

5 Contact: Yehuda Ben-Shahar, benshahary@wustl.edu, Department of Biology,

6 Washington University in St. Louis, St. Louis, MO 63130

7 


\begin{abstract}
Many insect species exhibit basal social behaviors such as aggregation, which play important roles in their feeding and mating ecologies. However, the evolutionary, genetic, and physiological mechanisms that regulate insect aggregation remain unknown for most species. Here, we used natural populations of Drosophila melanogaster to identify the genetic architecture that drives larval aggregation feeding behavior. By using quantitative and reverse genetic approaches, we have identified a complex neurogenetic network that plays a role in regulating the decision of larvae to feed in either solitude or as a group. Results from single gene, RNAi-knockdown experiments show that several of the identified genes represent key nodes in the genetic network that determines the level of aggregation while feeding. Furthermore, we show that a single noncoding SNP in the gene CG14205, a putative acyltransferase, is associated with both decreased mRNA expression and increased aggregate formation, which suggests that it has a specific role in inhibiting aggregation behavior. Our results identify, for the first time, the genetic components which interact to regulate naturally occurring levels of aggregation in D. melanogaster larvae.
\end{abstract}

Keywords: Drosophila melanogaster; fruit fly; vinegar fly; foraging; sociality 


\section{Introduction:}

29 Group formation is one of the simplest forms of social interaction exhibited by

30 individual animals. Yet, the genetic and physiological mechanisms underlying group

formation are largely unknown for most species. Drosophila melanogaster larvae form

simple cooperative group aggregates while feeding, which has been hypothesized to increase their fitness by providing defense against predation, as well as enabling individuals to communally digest food substrates more easily (Prokopy \& Roitberg, 2001; Sokolowski, 2010; Wu et al., 2003). Previous studies have suggested that in

Drosophila and several other insect species, the formation and maintenance of larval aggregation is primarily regulated by the chemosensory detection of aggregation pheromones, as well as other sensory modalities (Leonhardt et al., 2016; Louis \& de Polavieja, 2017; Rooke et al., 2020; Steiger \& Stok1, 2017; Symonds \& Wertheim, 2005; Thibert et al., 2016). Specifically, in Drosophila melanogaster, at least two pheromones produced by larvae have been shown to act as chemoattractants (Mast et al., 2014). However, the downstream neural and genetic pathways that regulate larval aggregation behavior remain largely unexplored. while feeding is likely regulated by the interplay between attractive and repulsive signals directly emitted by other conspecifics, or indirectly via feeding-related chemical changes of the consumed food. Indeed, it has been shown that food patch choice is influenced by the presence of other larvae, and the decision to choose one food patch over another is a function of group size (Durisko \& Dukas, 2013; Lihoreau et al., 2016)

50 and genetics (Allen et al., 2017; Fitzpatrick et al., 2007; Kaun, Hendel, et al., 2007;

51 Kaun, Riedl, et al., 2007). However, although some conserved peptidergic signaling pathways have been shown to regulate aggregation in Drosophila larvae (Wu et al., 
53 2003), most signals and downstream neuronal and genetic pathways that regulate group

54 size via attractive and repulsive signals, remain unknown. important not only for deciphering the biological principles that drive social decision making in general, but would provide insight into means of offsetting the economic impact of insect pests. To address this important question, we used the Drosophila Genetic Reference Panel (DGRP) (Mackay et al., 2012) to identify genetic variations associated with the extent of larval feeding aggregate size. By combining a genomewide, quantitative genetics approach with single gene manipulations, we have identified several key genes that contribute to group size in natural populations of Drosophila

63 larva. Our results highlight the utility of D. melanogaster for understanding the genetics

64 of group formation and provide several genetic targets for further research on this topic.

\section{Materials and Methods:}

66

\section{Animals}

All fly lines were reared on standard corn syrup-soy food (Achron Scientific), and kept under a $12 \mathrm{~h}: 12 \mathrm{~h}$ light:dark schedule at $25{ }^{\circ} \mathrm{C}$ and $60 \%$ humidity. Lines from the Drosophila Genetic Reference Panel (DGRP) (Mackay et al., 2012) used in this study are available from the Bloomington Drosophila Stock Center (BDSC, Bloomington, IN). UAS-RNAi lines and the elav-and tubulin-GAL4 lines were from either the Bloomington Drosophila Stock Center or the Vienna Drosophila Resource Center (VDRC) (Dietzl et al., 2007; Perkins et al., 2015). All fly lines used in this study, along with their stock numbers and genotypes, are listed in Table S1. 


\section{Larval aggregation assays}

76 Larval aggregation was assayed as follows. Approximately 30, second/third instar

77 larvae were collected from standard vials using a $15 \%$ sucrose solution (w/v). Larvae

78 were placed onto the center of a $60 \mathrm{~mm}$ petri dish containing $20 \%$ apple juice $(\mathrm{v} / \mathrm{v})$ and $1 \%$ agar (w/v) en masse and allowed to roam the plate freely for 15 minutes.

Subsequently, a picture of the plate was taken (Figure 1A), and the fraction of aggregating larvae was calculated as described below. All behavioral assays were conducted at $25{ }^{\circ} \mathrm{C}$ and $70 \%$ humidity.

(i) in physical contact with one another and (ii) burrowing into the agar plate. To

calculate the fraction of larvae that were aggregating, we summed the number of larvae

86 forming aggregates and divided it by the total number of larvae observable from the

87 picture taken at the end of the test period.

\section{Genome Wide Association Study}

89 A total of 4-9 behavioral assays were conducted for each DGRP line, and the mean proportion of aggregating larvae was used for comparison in a genome wide association study (GWAS). A linear regression model was run using the easyGWAS server (Grimm et al., 2017), with default parameters, to search for genotype by phenotype associations.

93 A total of 2,370,987 SNPs from each of 48 DGRP lines were included in the GWAS,

94 after filtering out any SNPs that were of the same genotype across all lines. Linkage 95 disequilibrium and minor allele frequencies (MAF) were calculated using PLINK 96 (Purcell et al., 2007). 
99 identified in the initial GWAS containing SNPs with a p-value of less than $10^{-4.5}$

100 (Warde-Farley et al., 2010). A gene was said to contain a SNP if the SNP occurred

101 within \pm 500 base pairs of its coding exons as annotated in the Drosophila reference

102 genome (version 5.57, FB2014 03). Subsequently, co-expression, co-localization,

103 shared protein domains, and protein-protein interactions were used to calculate the gene

104 interaction network, and up to 20 genes that were not identified as significant in the

105 GWAS were allowed to be added to the network. Genes added to the network were

106 selected such they maximized the number of connections between genes already present

107 in the network (Warde-Farley et al., 2010).

\section{Gene Ontology Analysis}

109 Genes containing SNPs with a p-value of less than $10^{-4.5}$ were screened for functionally-

110 enriched gene ontologies using the bioprofiling.de servers ProfCom framework

111 (Antonov et al., 2008). All genes included in the functional gene interaction network

112 were also screened for functionally enriched gene ontologies using GeneMANIA

113 (Warde-Farley et al., 2010). The gene interaction network included 20 additional genes

114 that did not contain significant SNPs; the GO terms found to be associated with this

115 network are therefore more general to a set of genes commonly found to interact with

116 one another, rather than those specifically identified in the GWAS.

\section{$117 \quad$ Real Time qRT-PCR}

118 mRNA was collected from groups of 10 whole larvae $(n=3-4$ replicates per line) using

119 Trizol (ThermoFisher) and reverse transcribed to cDNA using SuperScript III Reverse

120 Transcriptase (ThermoFisher). Sybr Green (ThermoFisher) was used to amplify and

121 quantify expression levels for all genes containing significant SNPs identified in the

122 GWAS. Expression values were calculated relative to the $r p 49$ control gene using the 
123 delta delta Ct method, as we have previously described (Hill et al., 2017; Lu et al.,

124 2012; Vernier et al., 2019). All qPCR primers used in this study are listed in Table S2.

\section{CG14205-GAL4 Transgenic Flies}

126 An approximately $3 \mathrm{kbp}(\mathrm{X}: 19590171-19593107)$ region of the $C G 14205$ promoter was

127 synthesized by Integrated DNA Technologies, Inc (IDT) and placed into the pUCIDT-

128 ampR plasmid (IDT). We subcloned this region into the pENTR-1A plasmid

129 (ThermoFisher) using KpnI and XhoI restriction sites on either side of the promoter,

130 and then used Gateway cloning (ThermoFisher) to move the promoter into the

131 pBPGAL4.2::p65 plasmid (Addgene \#26229) (Pfeiffer et al., 2010). This plasmid was

132 subsequently injected into BDSC line \#24483 (RainbowGene Inc.), and positive

133 offspring were identified and back-crossed into $w^{1118}$. The CG14205-GAL4 line was

134 crossed with $U A S-m C D 8:: G F P$ (BDSC \#32188) and imaged in third-instar larvae.

\section{Results}

\section{Genetic variation underlying group formation}

137 As D. melanogaster larvae develop, they exhibit a gradual increase in aggregation

138 behavior (Wu et al., 2003). However, the overall genetic architecture that drives the

139 quantitative aspects of larval aggregation remains largely unknown. Therefore, to better

140 understand the genetics underlying aggregation, we screened 48 randomly chosen

141 isogenic wild type lines from the Drosophila Genetic Reference Panel (DGRP)

142 (Mackay et al., 2012) for levels of aggregation in third instar larvae and subsequently

143 performed a genome wide association study (GWAS) to look for genetic variation

144 associated with this phenotype. 
146 with some lines tending not to form any aggregates (termed "Low" lines) and other

147 lines containing as many as 40-60\% of aggregating larvae (termed "High" lines) (Figure

148 1). We then ran ANOVAs to search for genetic variation (SNPs) associated with the

149 mean fraction of aggregating larvae across lines (Shorter et al., 2015; Swarup et al.,

150 2013). A total of 2,370,987 ANOVAs were run for each unfiltered SNP in the 48 DGRP

151 lines analyzed, which uncovered 58 significant SNPs $\left(p<10^{-5}\right)$. Subsequently, 17

152 protein coding genes that fall within $500 \mathrm{bp}$ of these SNPs were further considered as

153 candidate genes that might be playing a role in larval aggregation decisions (Figure 2,

154 Table S3).

\section{The neurogenetic network of larval aggregation behavior}

156 To investigate whether specific genetic pathways might be playing a role in larval

157 aggregation decisions, we next used gene ontology (GO) analyses. Because our initial

158 conservative $\mathrm{p}<10^{-5}$ significance threshold yielded only 17 protein-coding genes that

159 might be causally associated with levels of aggregation, we used the less conservative

160 threshold of $p<10^{-4.5}$, which increased the number of candidate genes to 68 . This

161 analysis indicated that this gene list is enriched for the GO terms "Axon guidance"

162 (GO:0007411, $\mathrm{p}=0.01)$ and "Plasma membrane" (GO:0005886, $\mathrm{p}=0.01)$. To further

163 expand the analysed gene network, we next extended the empirically defined gene

164 network by using the following edges: co-expression, co-localization, shared protein

165 domains, and protein-protein interactions (Supplemental Figure 1A). GO analysis of the

166 extended gene list was still enriched for "Axon guidance"; however, four out of the top

167 six enriched GO terms are neural-tissue specific (Supplemental Figure 1B). Together,

168 these data suggest that at least some of the genetic variations we have identified impact 
169 population level phenotypic variations in aggregation decisions via neuronal functions.

\section{$170 \quad$ Genetic variations associated with $m R N A$ expression levels}

171 Single nucleotide polymorphisms falling within promoter and enhancer regions of a

172 protein coding gene often affect mRNA expression levels (Khurana et al., 2016; Nord \&

173 West, 2020; Visel et al., 2009). Since most of the SNPs we have identified in our

174 GWAS are either intronic or fall upstream of their associated genes (37/46; Table S3),

175 we next tested the hypothesis that some of the identified SNPs affect gene action via

176 their effects on mRNA expression levels. To test this hypothesis, we compared the

177 mRNA expression levels of each of the 17 candidate genes identified in our initial

178 conservative screen between the three phenotypically highest ("High") and three lowest

179 (“Low”) aggregating DGRP lines (Figure 3A, and B) by using real-time qRT-PCR

180 analyses. We found that at least one SNP (X:19488026) was significantly associated

181 with higher mRNA expression levels of its parent gene, CG14205, in all "low" lines

182 relative to all "high" lines (one-way ANOVA; $F(1,4)=13.43, p=0.02$ ) (Figure 3).

183 These results suggest that this specific SNP is playing a role in regulating the expression

184 or stability of the CG14205 mRNAs. The location of this SNP immediately downstream

185 of a predicted splice donor site in the annotated intron 5 of CG14205 (Figure 3C)

186 suggests that it may affect splicing and/ or stability of the pre-mRNA. Furthermore, we

187 found a significant interaction between CG14205 expression level and SNP genotype on

188 the levels of aggregation between High and Low lines (two-way ANOVA; $F(2,3)=$

$189403.3, p<0.01)$. As $C G 14205$ expression is significantly higher in Low lines than in

190 High lines, these data suggest that higher expression levels of CG14205 may reduce

191 aggregation in D. melanogaster larvae. 
Although the biological functions of $C G 14205$ are unknown, the protein is predicted to be membrane bound Acyltransferase 3 (IPR002656) that is related to the

194 Nose resistant-to-fluoxetine (NRF) protein family in C. elegans (Choy \& Thomas, 195 1999). Since several family members have been found to be expressed in the gut

196 epithelium of worms, it has been hypothesized that they may function as novel

197 transporters of lipophilic molecules (Choy et al., 2006). However, the specific

198 biochemical functions of these membrane-bound acyltransferases remain

199 uncharacterized. Nevertheless, previous studies in the moth Bombyx mori, have shown

200 that various acyltransferases are required for the synthesis of sex pheromones in moths

201 and other insects (Ding et al., 2016; Mengfang Du et al., 2015; M. Du et al., 2012).

202 Further, a quantitative trait locus (QTL) associated with intra- and interspecific

203 variations in sex pheromones in noctuid moths has been mapped to the regulation of a

204 gene containing a putative Acyltransferase 3 domain (Groot et al., 2013). Therefore, it is

205 possible that CG14205 plays a direct role in the synthesis of larval aggregation

206 pheromones in D. melanogaster.

\section{Candidate gene knockdown leads to altered levels of aggregation}

208 To further establish a causal role for the genes identified in our initial screen, we studied

209 the effects of neuronal-specific RNAi knockdown of each gene by using the pan-

210 neuronal elav-GAL4 driver. However, neuronal knockdown of five of the 17 genes we

211 examined (Vha36-1, $d s x-c 73 a$, pros, cindr, and CG45002) was lethal. Of the remaining

21212 genes, neuronal knockdown of knockdown of four of the genes (CG8187, CG14502,

213 CG32206, and $r n$ ) lead to higher levels of aggregation relative to controls (Figure 4A,

214 B, and C). These results suggest that the activity of these four genes affects aggregation

215 decisions in feeding larvae. 
217 aggregation levels. Given the strong association between the specific CG14205 alleles,

218 mRNA expression levels, and aggregation levels, we next tested whether genetic

219 variation in this specific gene affect aggregation decision via its action in non-neuronal

220 tissues by using the ubiquitous tubulin-GAL4 driver to knockdown CG14205 in all

221 tissues. As CG14205 mRNA is expressed to a greater extent in Low aggregating lines, we hypothesized that knocking down CG14205 should lead to increased levels of aggregation. Indeed, global CG14205 knockdown resulted in an increase in the fraction of larvae aggregating (one-tailed, Student's T-test, $p=0.025$; Figure 4D). These results suggest that CG14205 functions to suppress aggregation in D. melanogaster larvae via neuronal-independent signalling pathways in the larval midgut. stimuli. It is possible that the CG14205 gene is responsible for the biosynthesis or release of a sensory stimulus which inhibits larvae from interacting with one another and forming groups. This hypothesis is consistent with the fact that CG14205 is required in non-neuronal cells for maintaining normal levels of larval aggregation

234 (compared to controls). Further, mining the FlyExpress and Flygut databases revealed 235 that the expression of CG14205 is enriched in enterocytes in the larval midgut (Buchon 236 et al., 2013; Celniker et al., 2009) (Figure 5A-B). This expression pattern was further

237 confirmed by imaging the transgenic expression of GFP under the control of the 238 CG14205 promoter, which revealed strong expression in the most proximal and distal 239 parts of the midgut (Figure 5C-H). Together, these results suggest that $C G 14205$ plays a 
240 role in the synthesis or release, rather than detection, of an inhibitory molecule

241 regulating aggregation.

\section{Discussion}

243 It is often assumed that group and social behaviors arise via complex interactions

244 between many genes. Here, we have used an unbiased behavioral quantitative genetic

245 screen to identify population-level natural genetic variations that underlie aggregation in

246 D. melanogaster larvae. As expected, our analysis revealed that the decision of

247 individuals on whether to aggregate with other conspecifics is likely depended on a

248 complex genetic network that acts in both neuronal and non-neuronal tissues.

249 Furthermore, by using in vivo genetic manipulations, we show that at the population

250 level, both qualitative and quantitative variations could be causally associated with the

251 overall observed behavioral variations between individuals. However, whether the

252 specific identified genes exert their impact on aggregation via a common pathway, and

253 the exact cellular and physiological processes affected by these genes, remain unknown.

254 Specifically, we found that quantitative expression variations across different

255 alleles of the CG14205 gene, which encodes a putative acetyl transferase, are strongly

256 associated with larval aggregation while feeding; DGRP lines that exhibit low levels of

257 aggregation express higher levels of $C G 14205$ transcripts relative to those that display

258 high levels of aggregation (Figure 3B). These data suggest that the activity of CG14205

259 inhibits the formation of larval aggregates. While the mechanism regulating this

260 variation in transcript levels is not known, the SNP identified in our initial GWAS

261 screen is adjacent to a predicted intronic splice donor site (Figure 3C), which may affect

262 mRNA splicing and/ or stability via posttranscriptional processes. How CG14205

263 activity in the gut might regulate larval aggregation remains unknown. Although our

264 RNAi knockdown studies indicate that CG14205 is not specifically required in neurons, 
265 it remains a possibility that it influences larval behavior via its action in glia or the

266 endocrine system. Alternatively, this gene could be required for the production of a

267 chemical signal that modulates larval aggregation decisions via the enzymatic

268 modification of gut metabolites (Blomquist et al., 2010; Chiu et al., 2019; Hunt \&

269 Borden, 1990).

270 Recent studies have identified both specific chemical cues — pheromones - and

271 receptors to be required for directing aggregation behaviors in D. melanogaster larvae

272 (Mast et al., 2014). Although most of what is known about pheromone synthesis in

273 Drosophila and other insects relates to cuticular hydrocarbons production by fat-body

274 cells and the oenocytes (Makki et al., 2014; Wicker-Thomas et al., 2015; Zelle et al.,

275 2019), our data indicate that gut derived metabolites can also possibley act as

276 pheromones in Drosophila. The possible contribution of CG14205 to pheromone

277 synthesis is further supported by previous findings about the contribution of

278 acyltransferases to pheromonal signalling in other insect species (Ding et al., 2016;

279 Zhang et al., 2017). Therefore, it is possible that this enzyme functions in the production

280 of some inhibitory chemical cues that Drosophila larvae are responsive to during

281 feeding.

Previous studies by us and others have shown that pheromone-driven social

283 interactions in Drosophila and other insects often require the balancing action of both

284 attractive and repulsive cues (Allison \& Cardé, 2016; Ben-Shahar et al., 2010;

285 Blomquist \& Vogt, 2003; Lu et al., 2012; Lu et al., 2014; McKinney et al., 2015; Zelle

286 et al., 2019). However, in our study, the knockdown of all identified candidate genes

287 leads to increased levels of larval aggregation, which suggest that the primary

288 contributions of these genes are to suppression of aggregation. One possible

289 interpretation of these data is that in natural populations of D. melanogaster, it may be 
290 that it is more beneficial for larvae to supress aggregation as a function of density to

291 maximize larval fitness. Another non-mutually exclusive explanation might be that our

292 lab assay conditions, and the specific behavioral paradigm used, biased our screen

293 towards the identification of genes whose role contributes specifically to the

294 suppression of larval aggregation.

295 Nevertheless, our study has uncovered several novel genes involved in directing

296 social aggregation while feeding in Drosophila larvae. Although we do not know yet

297 the specific molecular and cellular mechanisms by which any of these genes affect

298 larval feeding behaviors, our data further indicate that natural genetic polymorphisms

299 affect larval social feeding behaviors via both neuronal and non-neuronal pathways

300 (Allen et al., 2017; Anreiter et al., 2017; Sokolowski, 2010).

301 Disclosure statement

302 The authors declare there are no conflicts of interest.

303 Funding

304 This work was supported by grants from the National Science Foundation to YB-S

305 [NSF-IOS 1322783, NSF-IOS 1754264, and NSF-DBI 1707221]

\section{References}

307 Allen, A. M., Anreiter, I., Neville, M. C., \& Sokolowski, M. B. (2017). Feeding-Related Traits Are Affected by Dosage of the foraging Gene in Drosophila melanogaster. Genetics, 205(2), 761-773. https://doi.org/10.1534/genetics.116.197939

Allison, J. D., \& Cardé, R. T. (2016). Pheromone communication in moths : evolution, behavior, and application [still image]. University of California Press. https://doi.org/40026787697

Anreiter, I., Kramer, J., \& Sokolowski, M. B. (2017). Epigenetic mechanisms modulate differences in Drosophila foraging behavior. PNAS.

Antonov, A. V., Schmidt, T., Wang, Y., \& Mewes, H. W. (2008). ProfCom: a web tool for profiling the complex functionality of gene groups identified from high- 

https://doi.org/10.1093/nar/gkn239

Ben-Shahar, Y., Lu, B., Collier, D. M., Snyder, P. M., Schnizler, M., \& Welsh, M. J. (2010). The Drosophila gene CheB42a is a novel modifier of Deg/ENaC channel function. PLoS One, 5(2), e9395. https://doi.org/10.1371/journal.pone.0009395

Blomquist, G. J., Figueroa-Teran, R., Aw, M., Song, M., Gorzalski, A., Abbott, N. L., Chang, E., \& Tittiger, C. (2010). Pheromone production in bark beetles. Insect Biochem Mol Biol, 40(10), 699-712. https://doi.org/10.1016/j.ibmb.2010.07.013

Blomquist, G. J., \& Vogt, R. (2003). Insect pheromone biochemistry and molecular biology the biosynthesis and detection of pheromones and plant volatiles. Elsevier/Academic Press,

Buchon, N., Osman, D., David, F. P., Fang, H. Y., Boquete, J. P., Deplancke, B., \& Lemaitre, B. (2013). Morphological and molecular characterization of adult midgut compartmentalization in Drosophila. Cell Rep, 3(5), 1725-1738. https://doi.org/10.1016/j.celrep.2013.04.001

Celniker, S. E., Dillon, L. A., Gerstein, M. B., Gunsalus, K. C., Henikoff, S., Karpen, G. H., Kellis, M., Lai, E. C., Lieb, J. D., MacAlpine, D. M., Micklem, G., Piano, F., Snyder, M., Stein, L., White, K. P., \& Waterston, R. H. (2009). Unlocking the secrets of the genome. Nature, 459(7249), 927-930. https://doi.org/459927a [pii] $10.1038 / 459927 \mathrm{a}$

Chiu, C. C., Keeling, C. I., \& Bohlmann, J. (2019). The cytochrome P450 CYP6DE1 catalyzes the conversion of $\alpha$-pinene into the mountain pine beetle aggregation pheromone trans-verbenol. Sci Rep, 9(1), 1477. https://doi.org/10.1038/s41598$\underline{018-38047-8}$

Choy, R. K., Kemner, J. M., \& Thomas, J. H. (2006). Fluoxetine-resistance genes in Caenorhabditis elegans function in the intestine and may act in drug transport. Genetics, 172(2), 885-892. https://doi.org/10.1534/genetics.103.024869

Choy, R. K., \& Thomas, J. H. (1999). Fluoxetine-resistant mutants in C. elegans define a novel family of transmembrane proteins. Mol Cell, 4(2), 143-152. https://doi.org/10.1016/s1097-2765(00)80362-7

Dietzl, G., Chen, D., Schnorrer, F., Su, K. C., Barinova, Y., Fellner, M., Gasser, B., Kinsey, K., Oppel, S., Scheiblauer, S., Couto, A., Marra, V., Keleman, K., \& Dickson, B. J. (2007). A genome-wide transgenic RNAi library for conditional gene inactivation in Drosophila. Nature, 448(7150), 151-156. http://www.ncbi.nlm.nih.gov/entrez/query.fcgi? $\mathrm{cmd}=$ Retrieve $\& d b=$ PubMed\&do $\mathrm{pt}=$ Citation\&list uids $=17625558$

Ding, B. J., Lager, I., Bansal, S., Durrett, T. P., Stymne, S., \& Lofstedt, C. (2016). The Yeast ATF1 Acetyltransferase Efficiently Acetylates Insect Pheromone Alcohols: Implications for the Biological Production of Moth Pheromones. Lipids, 51(4), 469-475. https://doi.org/10.1007/s11745-016-4122-4

Du, M., Liu, X., Liu, X., Yin, X., Han, S., Song, Q., \& An, S. (2015). Glycerol-3phosphate $\mathrm{O}$-acyltransferase is required for PBAN-induced sex pheromone biosynthesis in Bombyx mori. Sci Rep, 5(1), 8110. https://doi.org/10.1038/srep08110

Du, M., Zhang, S., Zhu, B., Yin, X., \& An, S. (2012). Identification of a diacylglycerol acyltransferase 2 gene involved in pheromone biosynthesis activating neuropeptide stimulated pheromone production in Bombyx mori. J Insect Physiol, 58(5), 699-703. https://doi.org/10.1016/j.jinsphys.2012.02.002 
Durisko, Z., \& Dukas, R. (2013). Attraction to and learning from social cues in fruitfly larvae. Proc Biol Sci, 280(1767), 20131398. https://doi.org/10.1098/rspb.2013.1398

Fitzpatrick, M. J., Feder, E., Rowe, L., \& Sokolowski, M. B. (2007). Maintaining a behaviour polymorphism by frequency-dependent selection on a single gene. Nature, 447(7141), 210-212. https://doi.org/nature05764 [pii]10.1038/nature05764

Grimm, D. G., Roqueiro, D., Salome, P. A., Kleeberger, S., Greshake, B., Zhu, W., Liu, C., Lippert, C., Stegle, O., Scholkopf, B., Weigel, D., \& Borgwardt, K. M. (2017). easyGWAS: A Cloud-Based Platform for Comparing the Results of Genome-Wide Association Studies. Plant Cell, 29(1), 5-19. https://doi.org/10.1105/tpc.16.00551

Groot, A. T., Staudacher, H., Barthel, A., Inglis, O., Schofl, G., Santangelo, R. G., Gebauer-Jung, S., Vogel, H., Emerson, J., Schal, C., Heckel, D. G., \& Gould, F. (2013). One quantitative trait locus for intra- and interspecific variation in a sex pheromone. Mol Ecol, 22(4), 1065-1080. https://doi.org/10.1111/mec.12171

Hill, A., Zheng, X., Li, X., McKinney, R., Dickman, D., \& Ben-Shahar, Y. (2017). The Drosophila Postsynaptic DEG/ENaC Channel ppk29 Contributes to Excitatory Neurotransmission. J Neurosci, 37(12), 3171-3180. https://doi.org/10.1523/JNEUROSCI.3850-16.2017

Hunt, D. W., \& Borden, J. H. (1990). Conversion of verbenols to verbenone by yeasts isolated fromDendroctonus ponderosae (Coleoptera: Scolytidae). J Chem Ecol, 16(4), 1385-1397. https://doi.org/10.1007/BF01021034

Kaun, K. R., Hendel, T., Gerber, B., \& Sokolowski, M. B. (2007). Natural variation in Drosophila larval reward learning and memory due to a cGMP-dependent protein kinase. Learn Mem, 14(5), 342-349. https://doi.org/14/5/342 [pii]

10.1101/lm.505807

Kaun, K. R., Riedl, C. A., Chakaborty-Chatterjee, M., Belay, A. T., Douglas, S. J., Gibbs, A. G., \& Sokolowski, M. B. (2007). Natural variation in food acquisition mediated via a Drosophila cGMP-dependent protein kinase. J Exp Biol, $210(\mathrm{Pt}$ 20), 3547-3558. https://doi.org/210/20/3547 [pii]

$10.1242 /$ jeb.006924

Khurana, E., Fu, Y., Chakravarty, D., Demichelis, F., Rubin, M. A., \& Gerstein, M. (2016). Role of non-coding sequence variants in cancer. Nat Rev Genet, 17(2), 93-108. https://doi.org/10.1038/nrg.2015.17

Leonhardt, S. D., Menzel, F., Nehring, V., \& Schmitt, T. (2016). Ecology and Evolution of Communication in Social Insects. Cell, 164(6), 1277-1287. https://doi.org/10.1016/j.cell.2016.01.035

Lihoreau, M., Clarke, I. M., Buhl, J., Sumpter, D. J., \& Simpson, S. J. (2016). Collective selection of food patches in Drosophila. J Exp Biol, $219(\mathrm{Pt} 5), 668$ 675. https://doi.org/10.1242/jeb.127431

Louis, M., \& de Polavieja, G. (2017). Collective Behavior: Social Digging in Drosophila Larvae. Curr Biol, 27(18), R1010-R1012. https://doi.org/10.1016/j.cub.2017.08.023

Lu, B., LaMora, A., Sun, Y., Welsh, M. J., \& Ben-Shahar, Y. (2012). ppk23-Dependent chemosensory functions contribute to courtship behavior in Drosophila melanogaster. PLoS Genet, 8(3), e1002587. https://doi.org/10.1371/journal.pgen.1002587 
Lu, B., Zelle, K. M., Seltzer, R., Hefetz, A., \& Ben-Shahar, Y. (2014). Feminization of pheromone-sensing neurons affects mating decisions in Drosophila males. Biol Open, In Press(2), 152-160. https://doi.org/10.1242/bio.20147369

Mackay, T. F. C., Richards, S., Stone, E. A., Barbadilla, A., Ayroles, J. F., Zhu, D., Casillas, S., Han, Y., Magwire, M. M., \& Cridland, J. M. (2012). The Drosophila melanogaster Genetic Reference Panel. Nature, 482(7384), 173-178.

Makki, R., Cinnamon, E., \& Gould, A. P. (2014). The development and functions of oenocytes. Annu Rev Entomol, 59, 405-425. https://doi.org/10.1146/annurevento-011613-162056

Mast, J. D., De Moraes, C. M., Alborn, H. T., Lavis, L. D., \& Stern, D. L. (2014). Evolved differences in larval social behavior mediated by novel pheromones. Elife, 3, e04205. https://doi.org/10.7554/eLife.04205

McKinney, R. M., Vernier, C., \& Ben-Shahar, Y. (2015). The neural basis for insect pheromonal communication. Current Opinion in Insect Science, 12, 86-92. https://doi.org/http://dx.doi.org/10.1016/j.cois.2015.09.010

Nord, A. S., \& West, A. E. (2020). Neurobiological functions of transcriptional enhancers. Nat Neurosci, 23(1), 5-14. https://doi.org/10.1038/s41593-019-0538$\underline{5}$

Perkins, L. A., Holderbaum, L., Tao, R., Hu, Y., Sopko, R., McCall, K., Yang-Zhou, D., Flockhart, I., Binari, R., Shim, H.-S., Miller, A., Housden, A., Foos, M., Randkelv, S., Kelley, C., Namgyal, P., Villalta, C., Liu, L.-P., Jiang, X., HuanHuan, Q., Wang, X., Fujiyama, A., Toyoda, A., Ayers, K., Blum, A., Czech, B., Neumuller, R., Yan, D., Cavallaro, A., Hibbard, K., Hall, D., Cooley, L., Hannon, G. J., Lehmann, R., Parks, A., Mohr, S. E., Ueda, R., Kondo, S., Ni, J.Q., \& Perrimon, N. (2015). The Transgenic RNAi Project at Harvard Medical School: Resources and Validation. Genetics, 201(3), 843-852. https://doi.org/10.1534/genetics.115.180208

Pfeiffer, B. D., Ngo, T. T., Hibbard, K. L., Murphy, C., Jenett, A., Truman, J. W., \& Rubin, G. M. (2010). Refinement of tools for targeted gene expression in Drosophila. Genetics, 186(2), 735-755. https://doi.org/genetics.110.119917 [pii] 10.1534/genetics.110.119917

Prokopy, R. J., \& Roitberg, B. D. (2001). Joining and avoidance behavior in nonsocial insects. Annu Rev Entomol, 46, 631-665. https://doi.org/10.1146/annurev.ento.46.1.631

46/1/631 [pii]

Purcell, S., Neale, B., Todd-Brown, K., Thomas, L., Ferreira, M. A., Bender, D., Maller, J., Sklar, P., de Bakker, P. I., Daly, M. J., \& Sham, P. C. (2007). PLINK: a tool set for whole-genome association and population-based linkage analyses. $\mathrm{Am} J$ Hum Genet, 81(3), 559-575. https://doi.org/10.1086/519795

Rooke, R., Rasool, A., Schneider, J., \& Levine, J. D. (2020). Drosophila melanogaster behaviour changes in different social environments based on group size and density. Commun Biol, 3(1), 304. https://doi.org/10.1038/s42003-020-1024-Z

Shorter, J., Couch, C., Huang, W., Carbone, M. A., Peiffer, J., Anholt, R. R., \& Mackay, T. F. (2015). Genetic architecture of natural variation in Drosophila melanogaster aggressive behavior. Proc Natl Acad Sci U S A, 112(27), E35553563. https://doi.org/10.1073/pnas.1510104112

Sokolowski, M. B. (2010). Social interactions in "simple" model systems. Neuron, 65(6), 780-794. https://doi.org/10.1016/j.neuron.2010.03.007

Steiger, S., \& Stokl, J. (2017). Pheromones involved in insect parental care and family life. Curr Opin Insect Sci, 24, 89-95. https://doi.org/10.1016/j.cois.2017.09.006 
Swarup, S., Huang, W., Mackay, T. F., \& Anholt, R. R. (2013). Analysis of natural variation reveals neurogenetic networks for Drosophila olfactory behavior. Proc Natl Acad Sci U S A, 110(3), 1017-1022. https://doi.org/10.1073/pnas.1220168110

Symonds, M. R., \& Wertheim, B. (2005). The mode of evolution of aggregation pheromones in Drosophila species. J Evol Biol, 18(5), 1253-1263. https://doi.org/10.1111/j.1420-9101.2005.00971.x

Thibert, J., Farine, J. P., Cortot, J., \& Ferveur, J. F. (2016). Drosophila Food-Associated Pheromones: Effect of Experience, Genotype and Antibiotics on Larval Behavior. PLoS One, 11(3), e0151451. https://doi.org/10.1371/journal.pone.0151451

Vernier, C. L., Krupp, J. J., Marcus, K., Hefetz, A., Levine, J. D., \& Ben-Shahar, Y. (2019). The cuticular hydrocarbon profiles of honey bee workers develop via a socially-modulated innate process. Elife, 8 . https://doi.org/10.7554/eLife.41855

Visel, A., Rubin, E. M., \& Pennacchio, L. A. (2009). Genomic views of distant-acting enhancers. Nature, 461(7261), 199-205. https://doi.org/10.1038/nature08451

Warde-Farley, D., Donaldson, S. L., Comes, O., Zuberi, K., Badrawi, R., Chao, P., Franz, M., Grouios, C., Kazi, F., Lopes, C. T., Maitland, A., Mostafavi, S., Montojo, J., Shao, Q., Wright, G., Bader, G. D., \& Morris, Q. (2010). The GeneMANIA prediction server: biological network integration for gene prioritization and predicting gene function. Nucleic Acids Res, 38(Web Server issue), W214-220. https://doi.org/10.1093/nar/gkq537

Wicker-Thomas, C., Garrido, D., Bontonou, G., Napal, L., Mazuras, N., Denis, B., Rubin, T., Parvy, J. P., \& Montagne, J. (2015). Flexible origin of hydrocarbon/pheromone precursors in Drosophila melanogaster. J Lipid Res, 56(11), 2094-2101. https://doi.org/10.1194/jlr.M060368

Wu, Q., Wen, T., Lee, G., Park, J. H., Cai, H. N., \& Shen, P. (2003). Developmental control of foraging and social behavior by the Drosophila neuropeptide Y-like system. Neuron, 39(1), 147-161. https://doi.org/S0896627303003969 [pii]

Zelle, K., Vernier, C., Liang, X., Halloran, S., Millar, J., \& Ben-Shahar, Y. (2019). A pleiotropic chemoreceptor facilitates the coupling of pheromonal signal perception and production. bioRxiv, 124305. https://doi.org/10.1101/124305

Zhang, Y.-N., Zhang, L.-W., Chen, D.-S., Sun, L., Li, Z.-Q., Ye, Z.-F., Zheng, M.-Y., Li, J.-B., \& Zhu, X.-Y. (2017). Molecular identification of differential expression genes associated with sex pheromone biosynthesis in Spodoptera exigua. Molecular Genetics and Genomics, 292(4), 795-809. https://doi.org/10.1007/s00438-017-1307-3 
Figures

504 Figure 1: Variation in levels of aggregation between natural populations of

505 Drosophila. (A) An image of a DGRP line (Line 75) that showed low levels of

506 aggregation, and (B) an image of a DGRP line (Line 101) that showed high levels of

507 aggregation. White arrowheads point to groups of aggregating larvae. (C) Boxplots

508 showing the fraction of aggregating larvae for each of the 48 DGRP lines that were

509 included in the GWAS ( $\mathrm{n}=5-9$ replicates per line); outliers are shown with open circles.

510 DGRP lines with either low (Low) or high (High) levels of aggregation that were used

511 in subsequent analyses are labeled and shown in either light or dark blue, respectively.

512 Figure 2: A genome-wide association study identified 58 SNPs that were associated

513 with the extent of larval aggregation across DGRP lines. (A) Manhattan plot

514 showing transformed p-values for each of the SNPs included in the GWAS. SNPs with

515 a p-value less than 10-5 (shown by the dashed gray line) were retained for further

516 analysis and are outlined in red. (B) A higher resolution view of SNPs highlighted in

517 (A). (Top) Transformed p-values and (Middle) minor allele frequencies (MAFs) for

518 each of the retained SNPs. SNPs that fell within \pm 500 base pairs of the coding region of

519 a gene are labeled and highlighted together. Some SNPs fell within the coding region of

520 genes on both the plus and minus strand of DNA and are labelled accordingly. (Bottom)

521 Linkage disequilibrium matrix between all of the retained SNPs.

522 Figure 3: mRNA expression analysis of SNP-containing genes in lines with either

523 low or high levels of aggregation. (A) Relative mRNA expression levels for each of

524 the SNP-containing genes identified in the GWAS ( $\mathrm{n}=3-4$ replicates per line). Low

525 aggregating lines are shown in light blue, and High aggregating lines are shown in dark

526 blue. (B) Relative mRNA expression levels for the CG14205 gene. A significant 
association between SNP genotype and CG14205 mRNA expression was identified ( $p$ $<0.05$; one-way ANOVA), whereby Low aggregating lines had higher levels of expression than High aggregating lines. Note that Low and High lines segregated by genotype, as shown in (C). (C) Transformed p-values for associations (ANOVAs)

531 between specific SNP haplotypes and relative mRNA expression level of the gene

532 associated with that SNP. SNPs falling within the same gene are labeled and highlighted together, and SNPs which were significantly associated $(p<0.05)$ with mRNA expression of its gene are outlined in red. (D) Genetic architecture of the CG14205 gene and the DNA sequences surrounding the significantly associated SNP for each of the

536 Low and High DGRP lines. Note that the SNP, X:19488026 (denoted by a red arrow

537 head), falls just past the exon-intron boundary within intron 5 and is positioned to

538 potentially effect mRNA splicing.

Figure 4: Neuronal knockdown of some candidate genes leads to altered associated genes (UAS-RNAi lines from the Vienna Drosophila Resource Center).

542 Knockdown of CG8187 ( $\mathrm{n}=5-8, p<0.05), C G 14502(\mathrm{n}=8-9, p<0.05), C G 32206$

$543(\mathrm{n}=7-9, p<0.01)$, or $r n(\mathrm{n}=8-9, p<0.01)$ using elav-GAL4 lead to increased levels of 544 aggregation when compared to parental controls ( $n=6-19$, for all other lines). All 545 statistical comparisons used one-way ANOVA followed by a Tukeys HSD post-hoc 546 test. (B) Pan-neuronal RNAi-mediated knockdown of SNP-associated genes (UAS-

547 RNAi lines from the Bloomington TRiP collection). Knockdown of Dnah3 using elav-

548 GAL4 lead to a decrease in fraction of larvae aggregating $(\mathrm{n}=7-17, p<0.01)$, whereas 549 no other gene knockdowns were significantly different from control $(n=4-17)$. Pairwise

550 Students T-tests were run between each gene knockdown and control to look for 551 statistical significance, and p-values were adjusted for multiple comparisons using a 
552 Bonferroni correction. (C) TRiP-RNAi-mediated knockdown of CG14205 in neural

553 tissues, using the elav-GAL4 driver, did not lead to altered aggregation ( $\mathrm{n}=8$ per group,

$554 p>0.05$; onetailed Students T-test). (D) TRiP-RNAi-mediated knockdown of CG14205

555 in all tissues, using the tubulin-GAL4 driver, led to a significant increase in the fraction

556 of larvae aggregating compared to control $(\mathrm{n}=11-12, p=0.025$; one-tailed Students T-

557 test).

558 Figure 5: Interaction network of SNP-containing genes. (A) Expression levels of

559 CG14205 across larval tissues. Data were extracted from the FlyAtlas database. (B)

560 Expression levels of CG14205 across midgut cell types. ISC, Intestinal stem cells; EB,

561 Enteroblasts; EC, Enterocytes; EE, Enteroendocrine cells; VM, Visceral muscle. (C)

$562 C G 14205$ expression is restricted to an anterior and posterior regions of the larval

563 midgut. Image of an intact larva expressing GFP under the control of the CG14205

564 GAL4 line. (D-F) Image of $3^{\text {rd }}$ instar dissected gut: (D) Visible light image, (E) GFP

565 image, (D) Overlay. (G) High resolution confocal image of CG14205-expressing

566 anterior region (pink dashed box in E). Arrow shows GFP expression in stereotypical

567 enterocytes. (H) High resolution confocal image of CG14205-expressing posterior

568 region (blue dashed box in E). 


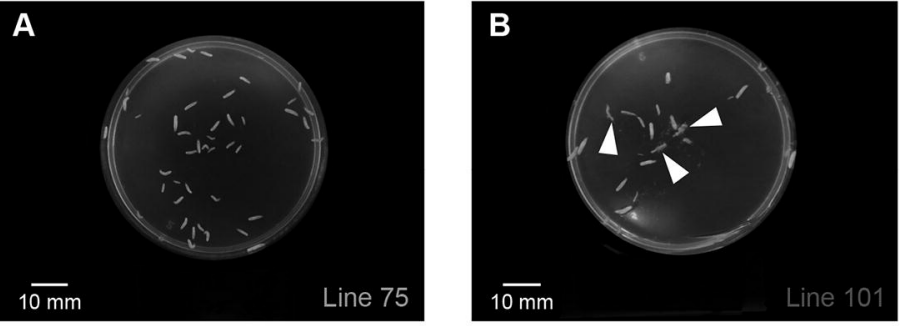

C

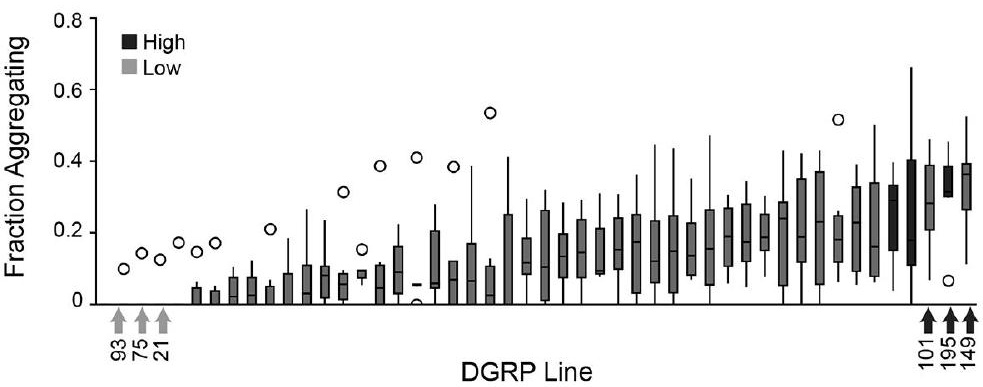


A

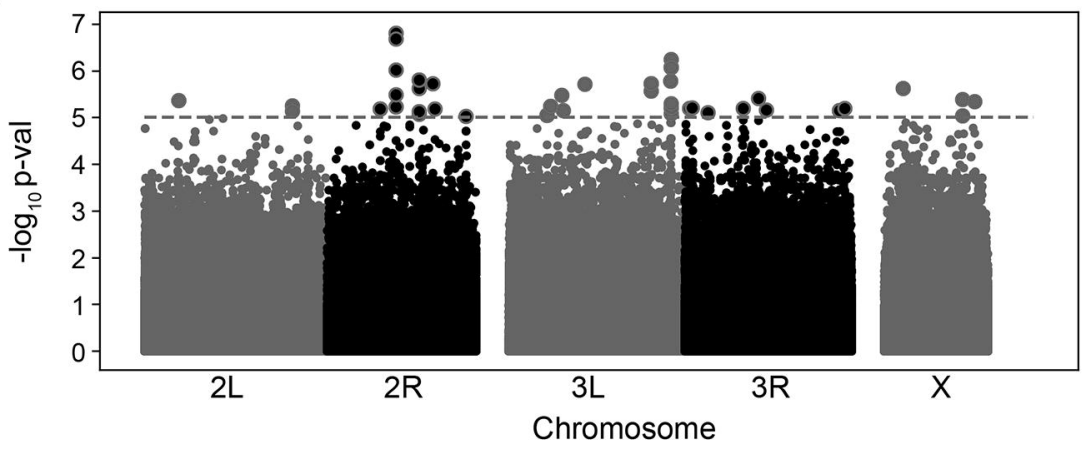

B

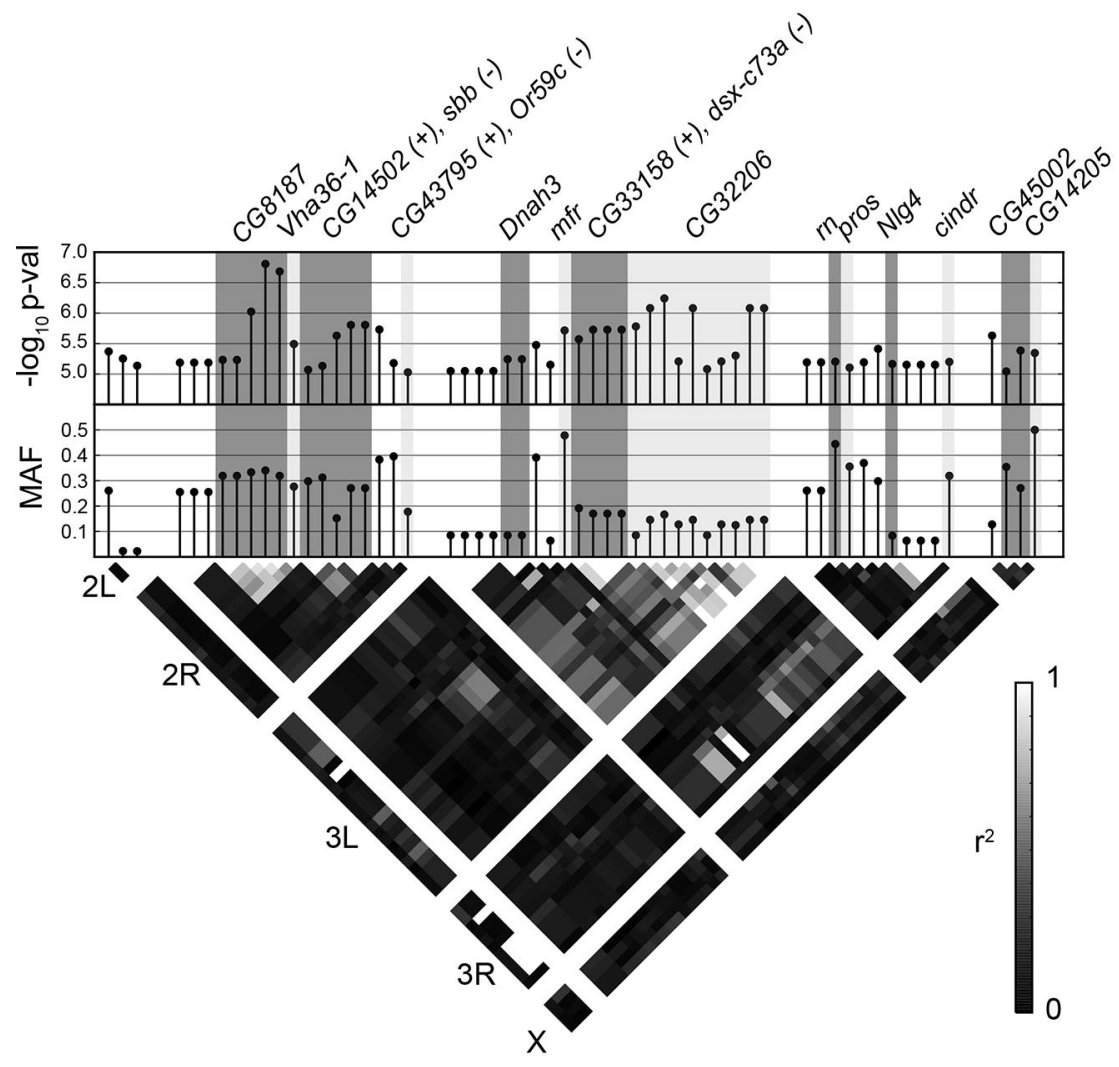




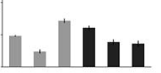

Vha36-1

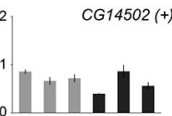

Or59c (-)
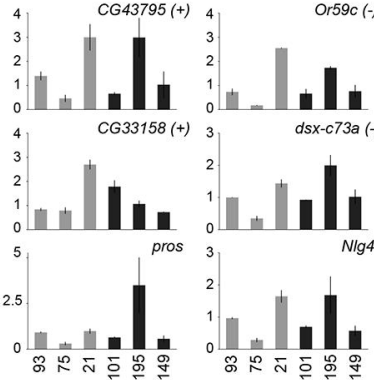

\section{Low}

$\square$ High

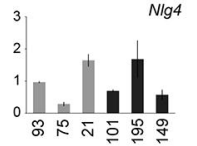

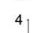

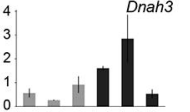

4

CG32206 3.
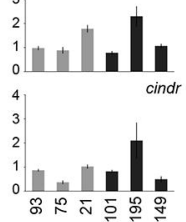

cindr

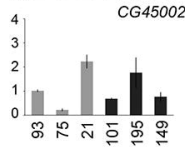

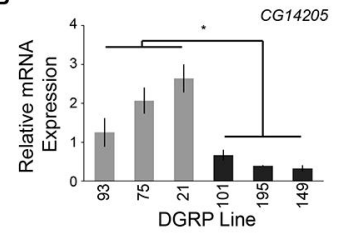

D

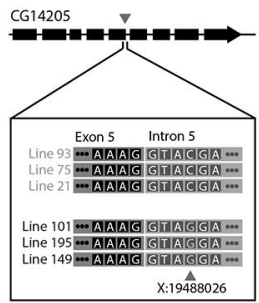

c

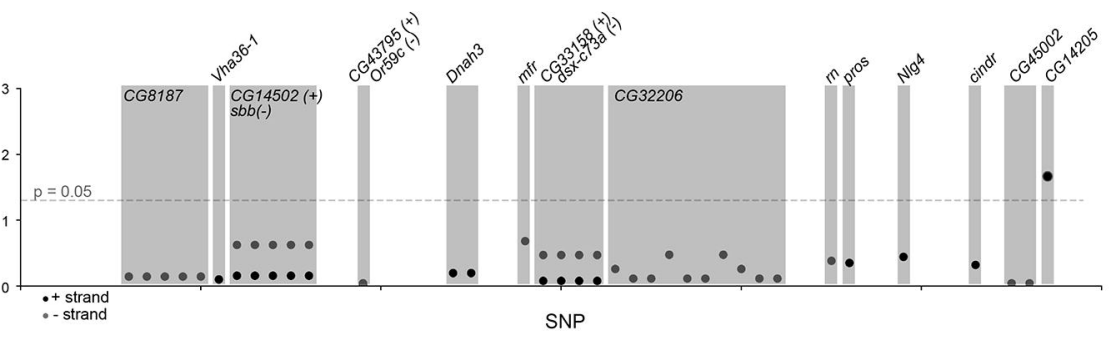



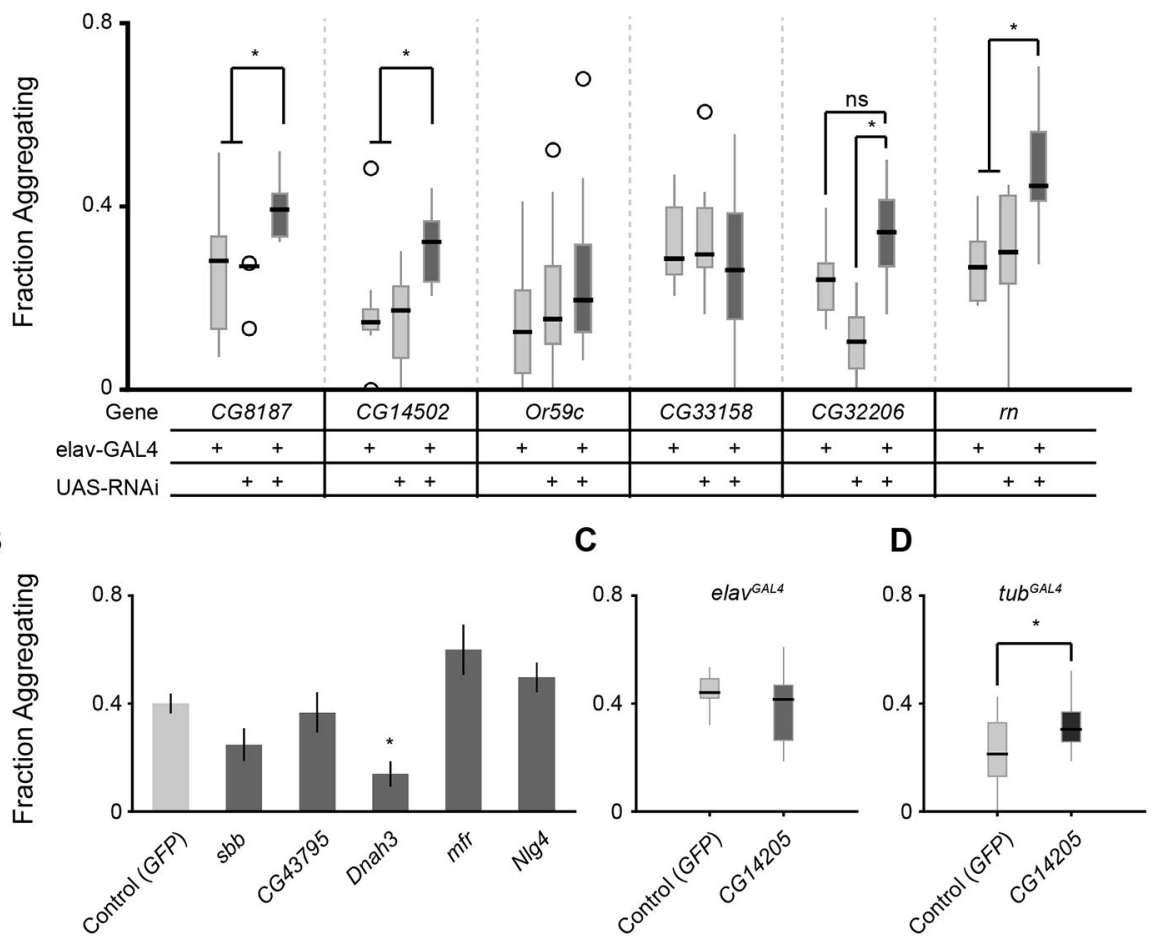


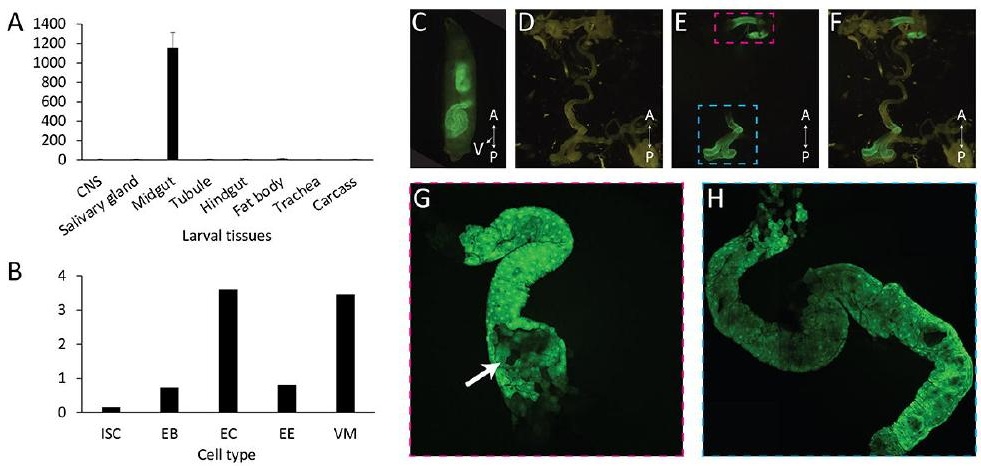

